\title{
Role Determination in an Aerial Dogfight
}

\author{
By G. J. OlsDer ${ }^{1}$ ) and J. V. BREAKWELL ${ }^{2}$ )
}

\begin{abstract}
A coplanar aerial dogfight is analyzed by assuming constant, not necessarily identical, speeds and individual maximum turning rates and lethal ranges. A combatant (A) is assumed to be victorious when his opponent (B) has been maneuvered into a relative position within A's lethal range and in the direction of A's velocity.

Three variables are required to define the instantaneous "state" of the game, namely the relative position (2) and the angle (1) between their velocities. A computer program has been constructed to divide the 3-dimensional region of possible initial (and subsequent) states into regions corresponding to victory by one or the other combatant, and, if the faster combatant has the smaller lethal range, a "no contest" region corresponding to escape by the faster combatant.

The critical separating surface (or surfaces) is composed of a number of pieces corresponding to initial conditions leading either to simultaneous kill or to "near miss" situations of one type or another. Optimal play is defined in the immediate neighborhood of the entire separating surface, guaranteeing victory (or escape) to one combatant or the other, depending on location on one side or the other of the separating surface.
\end{abstract}

\section{Introduction}

In a realistic aerial duel, or "dogfight", each combatant (player) wishes to destroy his opponent and to avoid destruction by his opponent. The dogfight problem consists essentially of two parts:

1. Given the initial conditions, determine the roles: Who pursues whom?

2. Given the roles, what are "time-optimal" strategies for each player?

We will be concerned only with the first question, which is certainly the more important one.

If we assume that a stalemate cannot arise, the "state space" (e.g., the space of initial conditions) must be divisible into regions corresponding to victory by one or the other player, or to escape by the faster player. This division was described, but only qualitatively, in the last sections of [BARON, Ho and KLEINMAN, 1970].

The division of the state-space will be discussed quantitatively in this paper for the idealized case in which both aircraft move in a single horizontal plane at constant speeds, with limited turn-rates, and with a lethal zone, in front of each aircraft, consisting of a line segment aligned with the velocity vector.

1) Supported by the Netherlands Organization for the Advancement of Pure Research, Contract No. S. 61-25. Now at Twente University of Technology Enschede: The Netherlands.

${ }^{2}$ ) G. J. OlsDer and J. V. BREAKWELl, Department of Aeronautics and Astronautics, Stanford University, Stanford, California 94305. 
A partial division of the state-space, using only the concept of "reachable sets", is obtained for a rather similar model in [CILETTI, MEIER and SALMON, 1973], where, however, the lethal zone took account of a finite rocket speed.

A discrete treatment, taking some account of incomplete information as well as the necessity of a finite lethal exposure time, is described in [FALCO and COHEN, 1973].

The state-space in our model is essentially three-dimensional: the instantaneous state may be defined, for example, by the two position coordinates of the second player in a coordinate system centered at the first player and aligned with his velocity vector, together with the angle $\theta$ between the two velocity vectors. It will further be assumed that one combatant, say player 1 , has both a larger speed and a smaller turn-rate capability than the other. A first attempt at the division of state-space in this model was made in an unpublished paper by A. W. MERZ, restricted, however, to the case of identical combatants which will be excluded in this paper.

The region $\mathscr{R}_{1}$ of the 3-dimensional state-space corresponding to victory by player 1 may be expected to be bounded by a surface consisting of a part of the surface $T_{1}$ of terminal states defining victory for player 1 , together with a "semipermeable" (see [ISAACS, 1964]) surface $\Sigma$ which prevents the state from leaving region $\mathscr{R}_{1}$ provided player 1 plays optimally in the neighborhood of $\Sigma$. The interior of region $\mathscr{R}_{1}$ does not contain any points of the terminal set $T_{2}$ corresponding to victory by player 2 , and thus, assuming that the contest ends in finite time, victory for player 1 is guaranteed for initial states inside $\mathscr{R}_{1}$. That portion of $T_{1}$ which forms part of the boundary of $\mathscr{R}_{1}$ is permeable only in the direction away from $\mathscr{R}_{1}$, provided player 2 plays optimally, which guarantees that no state outside of $\mathscr{R}_{1}$ can lead to victory by player 1 , provided that player 2 plays optimally in the vicinity of the boundary of $\mathscr{R}_{1}$. The situation is illustrated schematically in Fig. 1(a).

If we further assume that the lethal range of player 1 , the faster combatant, is less than that of player 2 , there will be an outer region of states from which player 1 can guarantee escape but from which he cannot guarantee victory. The region

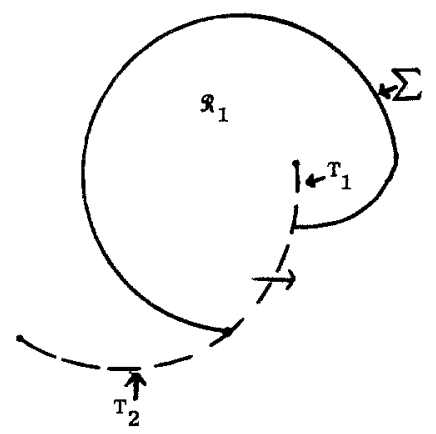

Fig. 1 (a) 
of state-space complementary to $\mathscr{R}_{1}$ will now be divided, by a second semipermeable surface $\Sigma^{\prime}$, into two regions $\mathscr{R}_{2}$ and $\mathscr{R}_{E}$, corresponding to victory by player 2 or escape by player 1 . The situation is illustrated in Figs. 1 (b) and 1 (c) the latter corresponding to a sufficiently large lethal range for player 2 .

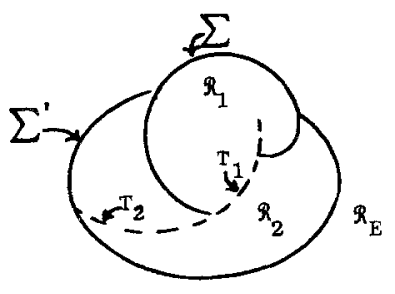

Fig. $1(b)$

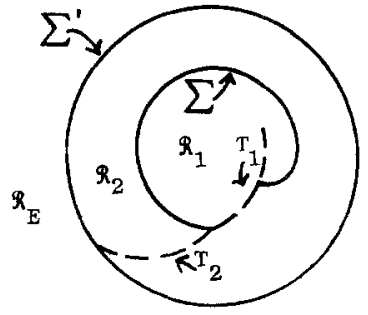

Fig. 1 (c)

Victory by player 2 in region $\mathscr{R}_{2}$ is guaranteed if we assume that no stalemate is possible, so that the surface $T_{2}$ is reached in finite time.

A method for the exact construction of the semi-permeable surface $\Sigma$ and $\Sigma^{\prime}$ will be discussed in detail in this paper. The surface $\Sigma^{\prime}$ will consist only of states from which optimal play by both players leads to a "near miss" in which player 1 either crosses player 2's lethal zone $T_{2}$, but only at its extremity, or else his path is tangent to $T_{2}$ without crossing it. The surface $\Sigma$ is more complex. It will contain pieces corresponding to "simultaneous kill", including collision, as well as pieces corresponding to states leading to near miss situations, which may be of two types: (i) the path in the state-space is tangent to $T_{1}$ or $T_{2}$ without crossing, (ii) player 2 crosses player 1's lethal zone at its extremity and avoids destruction because his angular velocity about player 1 exceeds player 1's maximum turn-rate. Case (ii) only arises if player 1's lethal range is sufficiently short.

The construction of $\Sigma$ and $\Sigma^{\prime}$ will be accomplished by the computation of the sections, in "planes" $\theta=$ constant, of all the possible pieces. The construction will be illustrated in the final section by a numerical example in which player 2's lethal range is so large that $\Sigma^{\prime}$ is of no interest.

\section{The General Form of the Analysis}

Two aircraft move in a single horizontal plane. Aircraft $i(i=1,2)$ has constant speed $v_{i}$, maximum angular velocity $\omega_{i}$, and a lethal range consisting of a line segment of length $l_{i}$ in the direction of its velocity vector. If player 1 crosses the lethal range of player 2 , or vice versa, the game ends.

We shall assume $v_{1}>v_{2}>0,0<\omega_{1}<\omega_{2}, 0<l_{1}<l_{2}$.

If $(x, y)$ are the position coordinates of player 2 relative to player 1 , with the $y$-axis aligned with player 1's velocity vector and the $x$-axis to the right of player 1 , 
and if $\theta$ is the angle measured clockwise from player 1's velocity vector to player 2's velocity vector, the equations of motion in this three dimensional "state space" are:

$$
\left.\begin{array}{l}
\dot{x}=-\omega_{1} u_{1} y+v_{2} \sin \theta \\
\dot{y}=\omega_{1} u_{1} x-v_{1}+v_{2} \cos \theta \\
\dot{\theta}=-\omega_{1} u_{1}+\omega_{2} u_{2}
\end{array}\right\}
$$

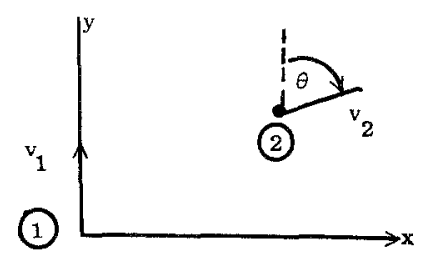

where the controls $u_{i}$ are the normalized turn-rates, i.e., $\left|u_{1}(t)\right| \leqq 1$, and

$$
\left.\begin{array}{l}
u_{i}=0 \Rightarrow \text { STRAIGHT LIKE MOTION } \\
u_{i}=1 \Rightarrow \text { FULL RIGHT TURN } \\
u_{i}=-1 \Rightarrow \text { FULL LEFT TURN }
\end{array}\right\} \text { IN “REAL" SPACE }
$$

Each portion of the semipermeable surface $\Sigma$, whether leading to simultaneous kill or to near miss, is obtainable by consideration of a particular local differential game, with a "terminal payoff" [ISAACS, 1964] defined in the immediate neighborhood of the simultaneous kill or near miss. If we adopt the convention that the local game has positive or negative value $V$ according to whether it ends in favor of player 2 or player 1, the "adjoint variables" $V_{x}, V_{y}, V_{\theta}$ and the optimal controls $u_{i}$ satisfy the "Main Equation":

$$
\underset{u_{2}}{\operatorname{Max}} \underset{u_{1}}{\operatorname{Min}}\left(V_{x} \dot{x}+V_{y} \dot{y}+V_{\theta} \dot{\theta}\right)=0
$$

which implies, in particular, the semi-permeability of the surface $V=0$ consisting of paths leading to simultaneous kill, or to near miss. The adjoint variables also satisfy:

$$
\left.\begin{array}{l}
\dot{V}_{x}=-V_{x} \frac{\partial \dot{x}}{\partial x}-V_{y} \frac{\partial \dot{y}}{\partial x}-V_{\theta} \frac{\partial \dot{\theta}}{\partial x}=-\omega_{1} u_{1} V_{y} \\
\dot{V}_{y}=\omega_{1} u_{1} V_{x} \\
\dot{V}_{\theta}=v_{2}\left(V_{y} \sin \theta-V_{x} \cos \theta\right)
\end{array}\right\}
$$

From (2.1) and (2.2) it follows that:

where

$$
u_{1}=\operatorname{sgn} A, \quad \text { if } \quad A \neq 0
$$

and

$$
A=y V_{x}-x V_{y}+V_{\theta}
$$

$$
u_{2}=\operatorname{sgn} V_{\theta}, \quad \text { if } \quad V_{\theta} \neq 0 .
$$


From (2.3) it follows that if $u_{1}$ remains constant for a duration at least $\tau$ prior to time $t_{f}$, then, apart from an unimportant constant multiplier, assumed henceforth to be unity, the adjoints at time $t=t_{f}-\tau$ satisfy:

$$
\left.\begin{array}{l}
V_{x}=\cos \left(\varphi-\omega_{1} u_{1} \tau\right) \\
V_{y}=\sin \left(\varphi-\omega_{1} u_{1} \tau\right)
\end{array}\right\} \quad \text { where }\left\{\begin{array}{l}
\cos \varphi=V_{x_{f}} \\
\sin \varphi=V_{y_{f}}
\end{array}\right.
$$

From (2.1), (2.3) and (2.6) it further follows that,

$$
\dot{V}_{\theta}=-v_{2} \cos \left(\theta+\varphi-\omega_{1} u_{1} \tau\right)=-v_{2} \cos \left(\theta_{f}+\varphi-\omega_{2} u_{2} \tau\right)
$$

so that, if $u_{2} \neq 0$,

$$
V_{\theta}=V_{\theta_{f}}-\frac{v_{2}}{\omega_{2} u_{2}}\left[\sin \left(\theta_{f}+\varphi-\omega_{2} u_{2} \tau\right)-\sin \left(\theta_{f}+\varphi\right)\right] .
$$

From (2.1), (2.3) and (2.6) we find that,

$$
\dot{A}=-v_{1} V_{x}
$$

so that, if $u_{1} \neq 0, \dot{A}=-\frac{v_{1}}{\omega_{1} u_{1}} \dot{V}_{y} \quad$ and,

$$
A=A_{f}+\frac{v_{1}}{\omega_{1} u_{1}}\left(V_{y_{f}}-V_{y}\right)
$$

Note that from (2.5) and (2.7) it follows that the only possible "singular arc" by player 2 , such that $V_{\theta}$ remains zero, is a straight-line motion $u_{2}=0$. Similarly, from (2.4), (2.9) and (2.3) it follows that the only possible singular arc by player 1 , such that $A$ remains zero, is a straight-line motion $u_{1}=0$.

The terminal values of the adjoints are obtained, for each differential game, in the following sections 3 and 4 . In each game, the terminal state $\left(x_{f}, y_{f}, \theta_{f}\right)$ together with the terminal "adjoint vector" $\left(V_{x_{f}}, V_{y_{f}}, V_{\theta_{f}}\right)$ are given in terms of a single parameter with a bounded range. Backwards construction of the paths is continued only as long as the switch-functions $A$ and $V_{\theta}$ have the signs appropriate to the optimal maneuvers indicated in Figs. 3 and 4 for the various local games.

The constructed paths for each local game thus provide a finite piece of surface in the state-space. The determination of $\Sigma$ from these pieces will be illustrated in section 5 by a numerical example. It will turn out that not all the pieces form part of $\Sigma$, and sometimes only a part of a piece forms part of $\Sigma$.

\section{The Local Games Associated with Simultaneous Kill}

There are essentially seven types of simultaneous kill, the corresponding maneuvers being shown in Fig. 3. Table 1 gives information on the terminal states and adjoints, from which the previous history of the switch-functions can be obtained and a maximum duration for each maneuver can be determined. 


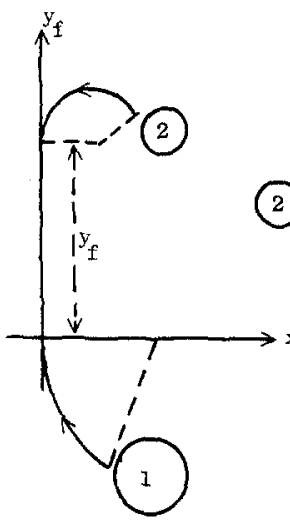

CASE:

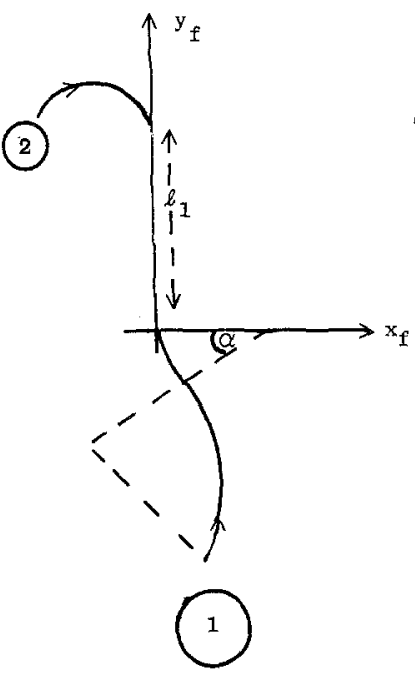

A $A$ S

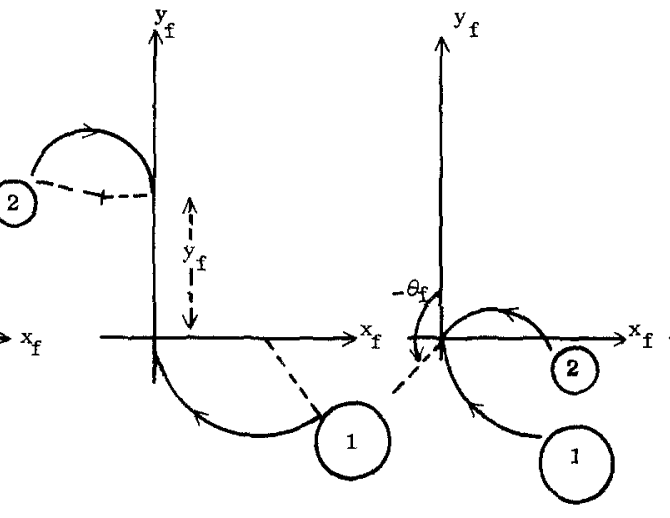

CASE 2

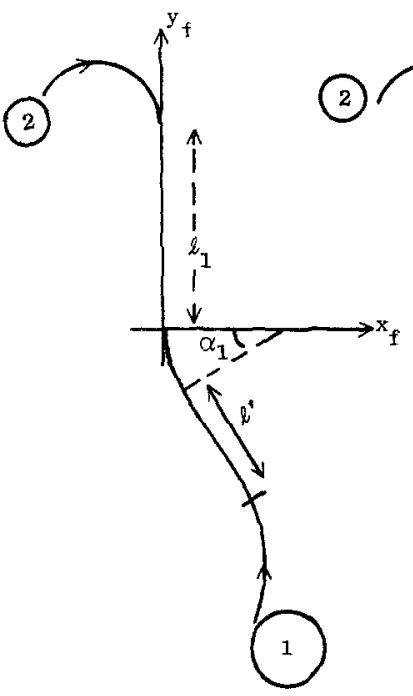

CASE 6

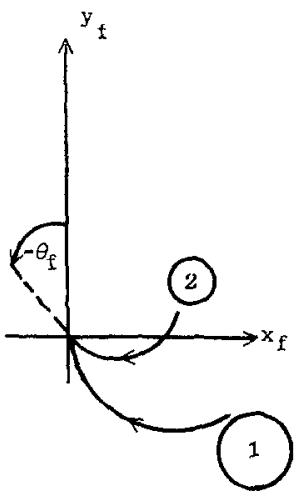

CASE 4

Fig. 3. Simultaneous Kill Maneuvers

In case 1 , player 1 turns right and player 2 turns left. The final state is $x_{f}=0$, $\theta_{f}=\pi$ and $0 \leqq y_{f} \leqq l_{1}$. Since $u_{1}=+1$, and $u_{2}=-1$, the duration $\tau$ is related to the initial $\theta$ by: $\theta=\pi+\left(\omega_{1}+\omega_{2}\right) \tau(\bmod 2 \pi)$. If $y_{f}<l_{1}$, the final adjoints clearly obey: $V_{x_{f}}>0, V_{y_{f}}=0$ and $V_{\theta_{f}}<0$, since, for example, a small displacement of player 2 to the right is in his favor, from which $V_{x_{f}}>0$. The other relations can be obtained similarly. Note that Eq. (2.2) gives a relation between $V_{x_{f}}, V_{y_{f}}$, and $V_{\theta_{f}}$. The duration $\tau$ must not exceed the first positive value at which $A$ or $V_{\theta}$ changes sign. If these never change sign, an obvious upper limit is given by $\omega_{2} \tau \leqq 2 \pi$, since player 2 would never find it advantageous to describe a complete turn. 
Table 1. Possibilities for simultaneous kill

\begin{tabular}{|c|c|c|c|c|c|c|c|c|c|}
\hline \multirow[t]{2}{*}{ Case } & \multicolumn{6}{|c|}{ End-conditions } & \multirow[t]{2}{*}{ Parameter and its limits } & \multicolumn{2}{|c|}{ Controls } \\
\hline & $x_{f}$ & $y_{f}$ & $\theta_{f}$ & $V_{x_{f}}$ & $V_{y_{f}}$ & $V_{\theta_{f}}$ & & $u_{1}$ & $u_{2}$ \\
\hline 1 & 0 & $y_{f}$ & $\pi$ & + & 0 & - & $0 \leqq y_{f} \leqq l_{1}$ & +1 & -1 \\
\hline 2 & 0 & $y_{f}$ & $\pi$ & + & 0 & + & $0 \leqq y_{f} \leqq l_{1}$ & +1 & +1 \\
\hline 3 & 0 & 0 & $\theta_{f}$ & + & - & 0 & $\operatorname{Cos}^{-1} \frac{v_{2}}{v_{1}} \leqq-\theta_{f} \leqq \pi$ & +1 & -1 \\
\hline 4 & 0 & 0 & $\theta_{f}$ & & - & 0 & $0 \leqq-\theta_{f} \leqq \operatorname{Cos}^{-1} \frac{v_{2}}{v_{1}}$ & +1 & +1 \\
\hline 5 & 0 & $l_{1}$ & $\pi$ & see & & & $0 \leqq \alpha \leqq \alpha_{1}$ & $-1 \rightarrow+1$ & +1 \\
\hline 6 & 0 & $l_{1}$ & $\pi$ & see & & & $0 \leqq l^{\prime} \leqq \frac{2 \pi v_{1}}{\omega_{2}}$ & $-1 \rightarrow 0 \rightarrow+1$ & +1 \\
\hline 7 & 0 & $l_{1}$ & $\pi$ & see & & & $0 \leqq l^{\prime} \leqq \frac{2 \pi v_{1}}{\omega_{2}}$ & $+1 \rightarrow 0 \rightarrow+1$ & \\
\hline
\end{tabular}

Case 2 is similar to case 1 , except that player 2 turns right, $u_{2}=+1$, and $V_{\theta_{f}}>0$.

Case 3 is a collision, with player 1 turning right and player 2 turning left. Clearly, $V_{\theta_{f}}=0, V_{x_{f}}>0, V_{y_{f}}<0$, so that $\cos \varphi>0, \sin \varphi<0$. From (2.1), (2.2) and (2.6), $v_{2} \sin \theta_{f} \cos \varphi-\left(v_{1}-v_{2} \cos \theta_{f}\right) \sin \varphi=0$, which now shows that $\sin \theta_{f}<0$ and determines $\varphi$ as a function of $\theta_{f}$. Since $-1=u_{2}=\operatorname{sgn} V_{\theta}, V_{\theta_{f}}=0$, we require $\left(\dot{V}_{\theta}\right)_{f} \geqq 0$. From (2.3) this requires $\sin \varphi \sin \theta_{f}-\cos \varphi \cos \theta_{f} \geqq 0$, which leads to $\cos \theta_{f} \geqq \frac{v_{2}}{v_{1}}$. The parameter $\theta_{f}$ thus satisfies $0 \leqq-\theta_{f} \leqq \operatorname{Cos}^{-1} \frac{v_{2}}{v_{1}}$. The duration $\tau$ is related to the initial $\theta$ by, $\theta=\theta_{f}+\left(\omega_{1}+\omega_{2}\right) \tau$. Note that case 3 with $\theta_{f}=\pi$ is identical to case 1 with $y_{f}=0$. The corresponding pieces of surface in state-space thus join together, but with discontinuous normal $\left(V_{x}, V_{y}, V_{\theta}\right)$.

Case 4 is similar to case 3 except that $u_{2}=+1,\left(\dot{V}_{\theta}\right)_{f} \leqq 0$, and hence $\cos \theta_{f} \leqq \frac{v_{2}}{v_{1}}$. The parameter $\theta_{f}$ thus satisfies $\operatorname{Cos}^{-1} \frac{v_{2}}{v_{1}} \leqq-\theta_{f} \leqq \pi$.

Interchange of left and right, reversing the signs of $x, \theta, u_{i}, V_{x}$ and $V_{\theta}$, leads to further cases not essentially different from cases 1 through 4 . These reflected cases will be denoted by $1^{\prime}$ through $4^{\prime}$.

Case 5 calls for an $S$-maneuver by player 1 to get within range of player 2 , who has the longer lethal range, before the latter, who is turning right, faces directly toward player 1 . If $\alpha$ is the turn-angle of the final turn by player 2, the duration $\tau_{1}$ of his previous reverse turn is related to the initial $\theta$ by:

$$
\theta=\pi-\omega_{2}\left(\frac{\alpha}{\omega_{1}}+\tau_{1}\right)+\alpha-\omega_{1} \tau_{1} \quad(\bmod 2 \pi)
$$


Since $A$ must change sign at $\tau=\frac{\alpha}{\omega_{1}}$, we obtain from (2.6) and (2.10):

$A_{f}+\frac{v_{1}}{\omega_{1}}[\sin \varphi-\sin (\varphi-\alpha)]=0$, and from (2.4): $A_{f}=l_{1} \cos \varphi+V_{\theta f}$. Also, from (2.2): $-\omega_{1} l_{1} \cos \varphi-\left(v_{1}+v_{2}\right) \sin \varphi+\left(\omega_{2}-\omega_{1}\right) V_{\theta_{f}}=0$. Elimination of $A_{f}$ and $V_{\theta_{f}}$ now yields:

$$
\omega_{2} l_{1} \cos \varphi+\left(v_{2}+\frac{\omega_{2} v_{1}}{\omega_{1}}\right) \sin \varphi=\frac{\omega_{2}-\omega_{1}}{\omega_{1}} v_{1} \sin (\varphi-\alpha),
$$

which suffices to determine $\varphi$ as a function of $\alpha$, the ambiguity in the quadrant of $\varphi$ being removed by the requirement that $A_{f} \geqq 0$. From (2.9) and (2.6) we require also that $\varphi-\alpha \geqq \frac{\pi}{2}$, in order that $\dot{A}$ be non-negative at player 1 's switch of turn direction. This leads in turn to the condition: $0 \leqq \alpha \leqq \alpha_{1}<\frac{\pi}{2}$, where $\alpha_{1}$ is the unique first quadrant solution of:

$$
\omega_{2} l_{1} \sin \alpha_{1}-\left(v_{2}+\frac{\omega_{2} v_{1}}{\omega_{1}}\right) \cos \alpha_{1}+\frac{\omega_{2}-\omega_{1}}{\omega_{1}} v_{1}=0
$$

The history of $V_{\theta}$ and $A$ prior to the switch is obtainable from (2.6), (2.7) and $(2.10)$ with $\varphi$ replaced by $\varphi-\alpha, \theta_{f}$ by $\pi-\left(\frac{\omega_{2}}{\omega_{1}}-1\right) \alpha, V_{\theta_{f}}$ by $V_{\theta_{f}}+$ $\frac{v_{2}}{\omega_{2}}\left[\sin \left(\varphi-\frac{\omega_{2}}{\omega_{1}} \alpha\right)-\sin \varphi\right]$, and $\tau$ being now measured backward from the switch. The duration $\tau_{1}$ of the initial turn by player 1 must not exceed the least positive $\tau$, if any, for which $V_{\theta}$ or $A$ change sign. Note that case 5 with $\alpha=0$ is identical to case $1^{\prime}$ with $y_{f}=l_{1}$.

Case 6 is an extension of the $S$-maneuver by player 1 , in which the final bend, with maximum angle $\alpha_{1}$, is preceded by a straight-line segment (singular arc) of length $l^{\prime}$, which is itself preceded by a left-turn of duration $\tau_{1}$ related to $l^{\prime}$ and the initial $\theta$ by:

$$
\theta=\pi-\omega_{2}\left(\frac{\alpha_{1}}{\omega_{1}}+\frac{l^{\prime}}{v_{1}}+\tau_{1}\right)+\alpha_{1}-\omega_{1} \tau_{1} \quad(\bmod 2 \pi)
$$

The values of $V_{x}, V_{y}$ and $A$ on the singular arc remain equal to 0,1 and 0 , so that $V_{\theta}=x$, which is easily obtainable as a function of $l^{\prime}$. The prior history of $V_{\theta}$ and $A$ is again easily constructible and limits on $\tau_{1}$ are thus obtainable.

Case 7 is similar to case 6 except that the singular arc is preceded by a right turn by player 1 , its duration $\tau_{1}$ being related to the initial $\theta$ by:

$$
\theta=\pi-\omega_{2}\left(\frac{\alpha}{\omega_{1}}+\frac{l^{\prime}}{v_{1}}+\tau_{1}\right)+\alpha_{1}+\omega_{1} \tau_{1} \quad(\bmod 2 \pi) .
$$

Again the left-right reflections of case 5 through 7 will be denoted by primes.

In all the cases described, the initial position $(x, y)$ of player 2 , in a coordinate system attached to player 1 , is obtainable in an elementary manner, and is expres- 
sible in terms of the initial $\theta$ and the parameter listed in Table 1. The resulting arcs in the "plane" $\theta$ are sections of semi-permeable pieces of surface which may form part of the desired separating surface $\Sigma$. We need, however, further semi-permeable pieces corresponding to local games associated with near misses. These will be discussed in the next section.

\section{The Local Games Associated with Near Miss}

There are essentially ten types of near miss, shown in Fig. 4. Table 2 gives the necessary information on the final states and adjoints.

Cases $a-d$ correspond to a right turn by player 1 in which player 2 avoids destruction because his angular motion about player 1 exceeds player 1's turn-rate when the separation decreases sufficiently. The general appearance of player 2's path in the coordinate system attached to player 1 is shown in Fig. 4(a).

The local game ends at $x_{f}=\dot{x}_{f}=0$, so that $\sin \theta_{f}=\frac{\omega_{1} y_{f}}{v_{2}}$, and clearly $V_{x_{f}}>0$, $V_{y_{f}}=V_{\theta_{f}}=0$, so that $\varphi=0$ and $A_{f}=y_{f}=\frac{v_{2}}{\omega_{1}} \sin \theta_{f}$. Cases $\left\{\begin{array}{l}\mathrm{a} \\ \mathrm{d}\end{array}\right.$ correspond

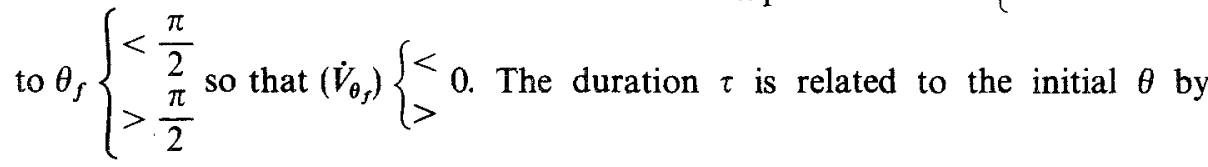
$\theta=\theta_{f}+\left(\omega_{1} \mp \omega_{2}\right) \tau(\bmod 2 \pi)$.

Cases $\mathrm{b}$ and $\mathrm{c}$ have $\theta_{f}=\frac{\pi}{2}$ and end with a singular arc by player 2 . If $l$ is the length of this arc, the adjoints on arrival at the singular arc are:

yielding

$$
V_{x}^{\prime}=\cos \frac{\omega_{1} l^{\prime}}{v_{2}}, \quad V_{y}^{\prime}=-\sin \frac{\omega_{1} l^{\prime}}{v_{2}}, \quad V_{\theta}^{\prime}=0,
$$

$$
A^{\prime}=\frac{v_{2}}{\omega_{1}}+\frac{v_{1}}{\omega_{1}} \sin \frac{\omega_{1} l^{\prime}}{v_{2}}, \text { and } \theta^{\prime}=\frac{\pi}{2}+\frac{\omega_{1} l^{\prime}}{v_{2}} .
$$

If $\tau$ is measured back from the start of the singular arc, the prior histories of $V_{\theta}$ and $A$ are, in cases $\left\{\begin{array}{l}\mathrm{b} \\ \mathrm{c}\end{array} V_{\theta}= \pm \frac{v_{2}}{\omega_{2}}\left(1-\cos \omega_{2} \tau\right)\right.$, which doesn't change sign, and

$$
A=\frac{v_{2}}{\omega_{1}}+\frac{v_{1}}{\omega_{1}} \sin \omega_{1}\left(\frac{l^{\prime}}{v_{2}}+\tau\right)
$$

which provides an upper limit $\frac{1}{\omega_{1}}\left(\pi+\operatorname{Sin}^{-1} \frac{v_{2}}{v_{2}}\right)$ on $\frac{l^{\prime}}{v_{2}}+\tau$, where the duration $\tau_{1}$ of the initial turn by player 1 is related to the $l^{\prime}$ and initial $\theta$ by:

$$
\theta=\frac{\pi}{2}+\omega_{1}\left(\frac{l^{\prime}}{v_{2}}+\tau_{1}\right) \mp \omega_{2} \tau_{1} \text {. }
$$



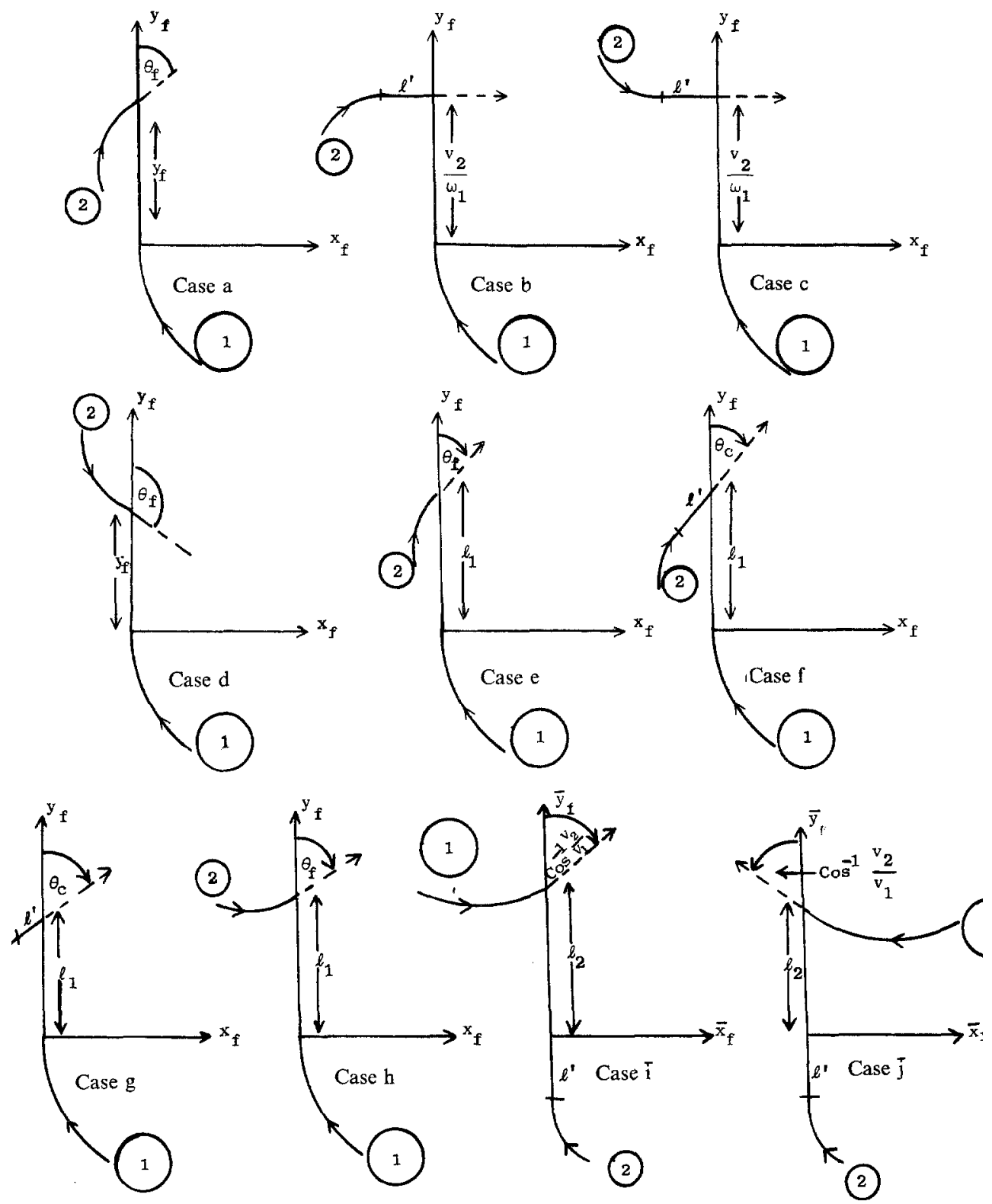

Fig. 4. Near Miss Maneuvers

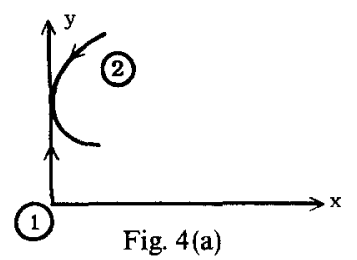


Role Determination in an Aerial Dogfight

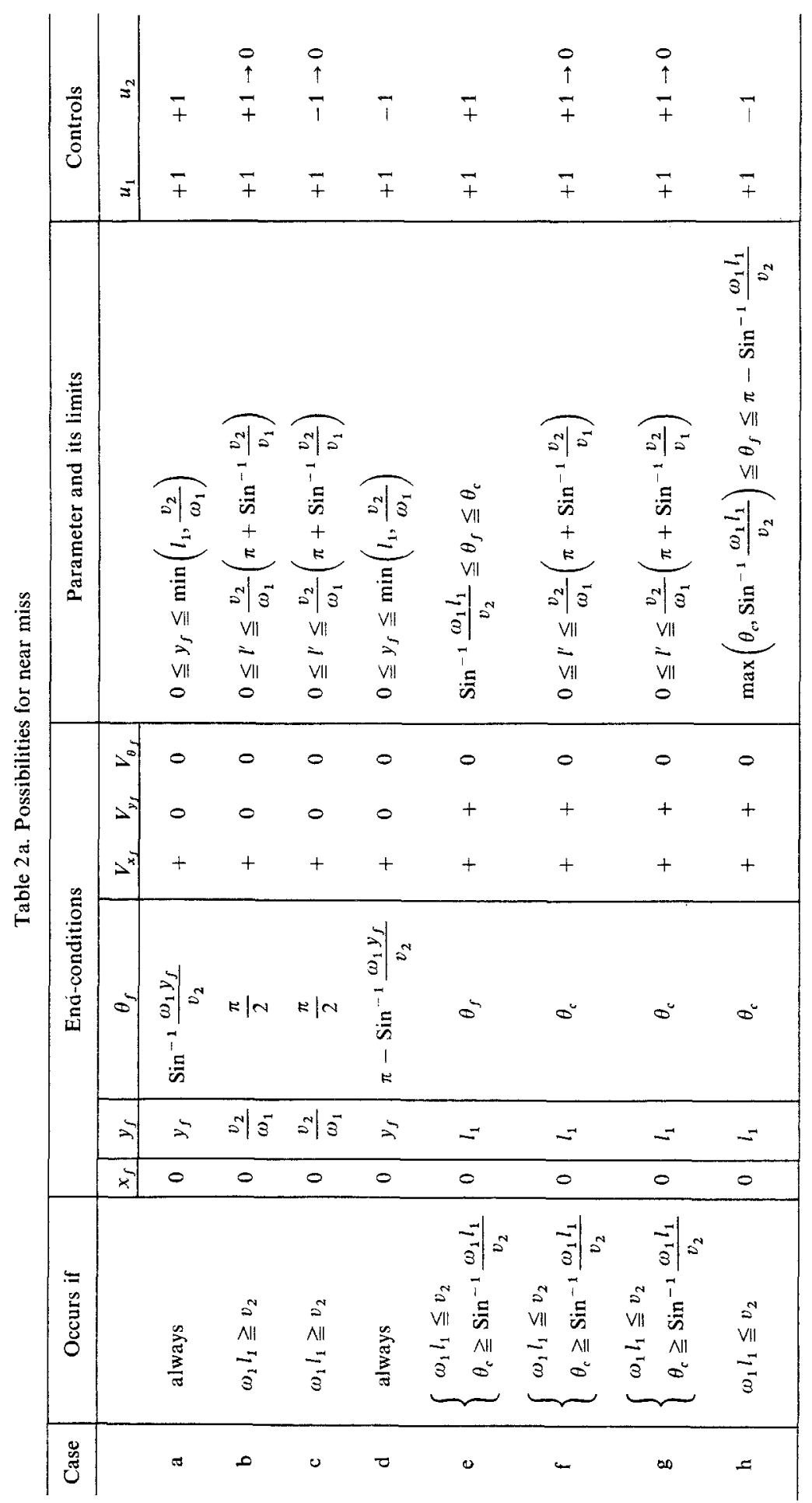


Table $2 \mathrm{~b}$. Possibilities for near miss

\begin{tabular}{|c|c|c|c|c|c|c|c|c|c|c|}
\hline \multirow[t]{2}{*}{ Case } & \multirow[t]{2}{*}{$\begin{array}{c}\text { Occurs } \\
\text { if }\end{array}$} & \multicolumn{6}{|c|}{ End-conditions } & \multirow[t]{2}{*}{$\begin{array}{l}\text { Parameter } \\
\text { and its limits }\end{array}$} & \multicolumn{2}{|c|}{ Controls } \\
\hline & & $\bar{x}_{f}$ & $\ddot{y}_{f}$ & $\bar{\theta}_{f}$ & $V_{x_{f}}$ & $V_{y_{f}}$ & $V_{\theta_{f}}$ & & $u_{2}$ & $u_{1}$ \\
\hline$\tilde{\mathrm{e}}$ & see text & 0 & $l_{2}$ & $\bar{\theta}_{f}$ & - & $?$ & 0 & $\bar{\theta}_{f}($ see text) & +1 & +1 \\
\hline $\bar{f}$ & see text & 0 & $l_{2}$ & $\bar{\theta}_{c}($ see text $)$ & - & ? & 0 & $0 \leqq l=\leqq \frac{2 \pi v_{1}}{\omega_{1}}$ & +1 & $+1 \rightarrow 0$ \\
\hline $\overrightarrow{\mathrm{g}}$ & see text & 0 & $l_{2}$ & $\partial_{c}($ see text $)$ & - & ? & 0 & $0 \leqq l^{\prime} \leqq \frac{2 \pi v_{1}}{\omega_{1}}$ & +1 & $-1 \rightarrow 0$ \\
\hline h & see text & 0 & $l_{2}$ & $\bar{\theta}_{f}$ & - & $?$ & 0 & $\bar{\theta}_{f}($ see text $)$ & +1 & -1 \\
\hline $\mathrm{i}$ & always & 0 & $l_{2}$ & $\operatorname{Cos}^{-1} \frac{v_{2}}{v_{1}}$ & 0 & - & 0 & $0 \leqq l^{\prime} \leqq \frac{2 \pi}{\omega_{1}}$ & $+1 \rightarrow 0$ & -1 \\
\hline $\mathrm{j}$ & always & 0 & $l_{2}$ & $-\operatorname{Cos}^{-1} \frac{v_{2}}{v_{1}}$ & 0 & - & 0 & $0 \leqq l \leqq \frac{2 \pi}{\omega_{1}}$ & $+1 \rightarrow 0$ & +1 \\
\hline
\end{tabular}

In addition to the left-right reflected cases $a^{\prime}-d^{\prime}$, we obtain eight more cases $\overline{\mathrm{a}}-\overline{\mathrm{d}}$ and $\overline{\mathrm{a}}^{\prime}-\overline{\mathrm{d}}^{\prime}$ by interchanging players 1 and 2 (and all appropriate subscripts). In cases $\bar{b}$ and $\bar{c}$, however, since $v_{2}<v_{1}, u_{2}=\operatorname{sgn}\left[\frac{v_{1}}{\omega_{2}}+\frac{v_{2}}{\omega_{2}} \sin \omega_{2}\left(\frac{l^{\prime}}{v_{1}}+\tau_{1}\right)\right]$ which remains positive, while $u_{1}= \pm \operatorname{sgn}\left(1-\cos \omega_{1} \tau\right)$, which doesn't change sign. In this case we impose the obvious limit $\frac{l^{\prime}}{v_{1}}+\tau_{1} \leqq \frac{2 \pi}{\omega_{2}}$.

If $\omega_{1} l_{1}<v_{2}$, cases $\mathrm{b}, \mathrm{c}$ do not arise, but we have cases $\mathrm{e}, \mathrm{f}, \mathrm{g}, \mathrm{h}$, in each of which $y_{f}=l_{1}$ and $\dot{x}_{f} \geqq 0$, so that $\sin \theta_{f} \geqq \frac{\omega_{1} l_{1}}{v_{2}}$. In each case, $V_{x_{f}} \geqq 0, V_{y_{f}} \geqq 0$, and $V_{\theta_{f}}=0$. With the aid of $(2.2)$ we obtain $\operatorname{sgn}\left(\dot{V}_{\theta}\right)_{f}=\operatorname{sgn}\left(v_{2}-v_{1} \cos \theta_{f}-\omega_{1} l_{1} \sin \theta_{f}\right)$. Now the equation $v_{1} \cos \theta+\omega_{1} l_{1} \sin \theta=v_{2}$ has a unique first quadrant solution $\theta_{c}$. Thus case e, with $u_{2}=+1$ arises only if $\theta_{c} \geqq \operatorname{Sin}^{-1} \frac{\omega_{1} l_{1}}{v_{2}}$ and $\operatorname{Sin}^{-1} \frac{\omega_{1} l_{1}}{v_{2}} \leqq \theta_{f} \leqq \theta_{c}$. Case h, with $u_{2}=-1$, arises if $\max \left(\theta_{c}, \operatorname{Sin}^{-1} \frac{\omega_{1} l_{1}}{v_{2}}\right) \leqq \theta_{f} \leqq \pi-\operatorname{Sin}^{-1} \frac{\omega_{1} l_{1}}{v_{2}}$. In both cases the value of $\varphi$ is determined by (2.2).

Cases $f, g$ with $\theta_{f}=\theta_{c}$, arise only if $\theta_{c} \geqq \operatorname{Sin}^{-1} \frac{\omega_{1} l_{1}}{v_{2}}$, and end, like cases $\mathrm{b}, \mathrm{c}$, with a singular arc by player 2 . If $l$ ' is the length of this arc, the adjoints on arrival at the singular arc are: $V_{x}^{\prime}=\cos \left(\frac{\pi}{2}-\theta_{c}-\frac{\omega_{1} l^{\prime}}{v_{2}}\right), V_{y}^{\prime}=\sin \left(\frac{\pi}{2}-\theta_{c} \theta \frac{\omega_{1} l^{\prime}}{v_{2}}\right)$, $V_{\theta}^{\prime}=0$, yielding $A^{\prime}=\frac{v_{2}}{\omega_{1}}-\frac{v_{1}}{\omega_{1}} \cos \left(\theta_{c}+\frac{\omega_{1} l^{\prime}}{v_{2}}\right)$ and $\theta^{\prime}=\theta_{c}+\frac{\omega_{1} l^{\prime}}{v_{2}}$. If $\tau$ is measured back from the start of the singular arc, the prior histories of $V_{\theta}$ and 
$A$ are, in cases $\left\{\begin{array}{l}\mathrm{f} \\ \mathrm{g}\end{array}: V_{\theta}= \pm \frac{v_{2}}{\omega_{2}}\left(1-\cos \omega_{2} \tau\right)\right.$, which doesn't change sign, and $A=\frac{v_{2}}{\omega_{1}}-\frac{v_{1}}{\omega_{1}} \cos \left[\theta_{c}+\omega_{1}\left(\frac{l^{\prime}}{v_{2}}+\tau\right)\right]$, which again provides an upper limit on $\frac{l^{\prime}}{v_{2}}+\tau_{1}$, where the duration $\tau_{1}$ of player 1 's initial turn is related to $l^{\prime}$ and the initial $\theta$ by: $\theta=\theta_{c}+\omega_{1}\left(\frac{l^{\prime}}{v_{2}}+\tau_{1}\right) \mp \omega_{2} \tau_{1}$.

The interchange of players in cases $e-h$ leads to further cases $\bar{e}-\bar{h}$, but the conditions under which they arise must be extended to include cases in which $\dot{\bar{y}}_{f} \geq 0$ and $\dot{\bar{x}}_{f}<0$ as well as cases in which $\dot{\bar{x}}_{f} \geqq 0,(\bar{x}, \bar{y})$ being the position of player 1 in a coordinate system attached to player 2 . This is because player 1 , the faster player, can avoid destruction by increasing the separation beyond $l_{2}$. In every case $V_{\bar{\theta}}=0, \bar{\theta},=-\theta$, being the angle clockwise from player 2 's velocity vector to player 1's velocity vector, and since $+1=u_{2}=-\operatorname{sgn} \bar{A}_{f}$ (player 2 is maximizing) with $\bar{A}=\bar{y} V_{\bar{x}}-\bar{x} V_{\bar{y}}+V_{\bar{\theta}}$, we see that $V_{\bar{x}_{f}} \leqq 0$. Also $u_{1}=-\operatorname{sgn} V_{\bar{\theta}_{f}}=$ $+\operatorname{sgn}\left(\dot{V}_{\bar{\theta}_{f}}\right)=\operatorname{sgn}\left[\left(v_{1} \cos \bar{\theta}_{f}-v_{2}\right)\left(v_{1}-v_{2} \cos \bar{\theta}_{f}-\omega_{2} l_{2} \sin \bar{\theta}_{f}\right)\right]$, with the aid of (2.2) and (2.3). Now the equation $v_{2} \cos \bar{\theta}+\omega_{2} l_{2} \sin \bar{\theta}=v_{1}$ has no solution if $\sqrt{v_{2}^{2}+\omega_{2}^{2} l_{2}^{2}}<v_{1}$, in which case $\cos ^{-1} \frac{v_{2}}{v_{1}}>\sin ^{-1} \frac{\omega_{2} l_{2}}{v_{1}}$, so that at least one of $\dot{\bar{x}}_{f}, \dot{\bar{y}}_{f}$ is positive, a unique first quadrant solution $\bar{\theta}_{c}$ less than $\cos ^{-1} \frac{v_{2}}{v_{1}}$ if $\omega_{2} l_{2}>v_{1}$, and the eqn. has two first quadrant solutions $\bar{\theta}_{c_{1}}, \bar{\theta}_{c_{2}}$, if $\omega_{2} l_{2}<v_{1}<$ $\sqrt{\omega_{2}^{2}+\omega_{2}^{2} l_{2}^{2}}$, in which case $\operatorname{Cos}^{-1} \frac{v_{2}}{v_{1}}<\operatorname{Sin}^{-1} \frac{\omega_{2} l_{2}}{v_{1}}$, with both $\dot{\bar{x}}_{f}$ and $\dot{\bar{y}}_{f}$ negative if $\bar{\theta}_{f}$ lies in this interval, and with $0<\bar{\theta}_{c_{1}}<\operatorname{Cos}^{-1} \frac{v_{2}}{v_{1}}<\operatorname{Sin}^{-1} \frac{\omega_{2} l_{2}}{v_{1}}<\bar{\theta}_{c_{2}}<\frac{\pi}{2}$. Case ē thus arises for,

$$
-\operatorname{Cos}^{-1} \frac{v_{2}}{v_{1}}<\theta_{f}<\left\{\begin{array}{l}
\operatorname{Cos}^{-1} \frac{v_{2}}{v_{1}}, \text { if } \sqrt{v_{2}^{2}+\omega_{2}^{2} l_{2}^{2}}<v_{1} \\
\tilde{\theta}_{c--}, \text { if } \omega_{2} l_{2}<v_{1}<\sqrt{v_{2}^{2}+\omega_{2}^{2} l_{2}^{2}} \\
\theta_{c}, \text { if } \omega_{2} l_{2}>v_{1}
\end{array}\right.
$$

and also, if $\omega_{2} l_{2}<v_{1}<\sqrt{v_{2}^{2}+\omega_{2}^{2} l_{2}^{2}}$, for $\operatorname{Sin}^{-1} \frac{\omega_{2} l_{2}}{v_{1}}<\bar{\theta}_{f}<\bar{\theta}_{c_{2}}$. Case $\bar{h}$ arises for $\operatorname{Cos}^{-1} \frac{v_{1}}{v_{1}}<\bar{\theta}_{f}<\pi-\operatorname{Sin}^{-1} \frac{\omega_{2} l_{2}}{v_{1}}$, if $\sqrt{v_{2}^{2}+\omega_{2}^{2} l_{2}^{2}}<v_{1}$, for $\bar{\theta}_{c_{1}}<\bar{\theta}_{f}<\operatorname{Cos}^{-1} \frac{v_{2}}{v_{1}}$ and $\bar{\theta}_{c_{2}}<\bar{\theta}_{f}<\pi-\operatorname{Sin}^{-1} \frac{\omega_{2} l_{2}}{v_{1}}$, if $\omega_{2} l_{2}<v_{1}<\sqrt{v_{2}^{2}+\omega_{2}^{2} l_{2}^{2}}$, and for $\bar{\theta}_{c}<\bar{\theta}_{f}<$ $\operatorname{Cos}^{-1} \frac{v_{2}}{v_{1}}$, if $\omega_{2} l_{2}>v_{1}$ 
Cases $\left\{\begin{array}{l}\overline{\mathrm{f}} \\ \overline{\mathrm{g}}\end{array}\right.$ arise, if $\omega_{2} l_{2}<v_{1}<\sqrt{v_{2}^{2}+\omega_{2}^{2} l_{2}^{2}}$, for $\bar{\theta}_{f}=\bar{\theta}_{c_{1}}$ or $\bar{\theta}_{c_{2}}$, and, if $\omega_{2} l_{2}>v_{1}$, for $\bar{\theta}_{f}=\bar{\theta}_{c}$. Here $V_{\bar{x}_{f}}=\cos \bar{\varphi}, V_{\bar{y}_{f}}=\sin \bar{\varphi}$, with $\bar{\varphi}=-\frac{\pi}{2}-\bar{\theta}_{f}$, and the switchfunctions prior to arrival at the singular arc are:

$-V_{\bar{\theta}}=\mp \frac{v_{1}}{\omega_{2}}\left(1-\cos \omega_{1} \tau\right)$ and $-\bar{A}=\frac{v_{1}}{\omega_{2}}-\frac{v_{2}}{\omega_{2}} \cos \left[\bar{\theta}_{f}+\omega_{2}\left(\frac{l^{\prime}}{v_{1}}+\tau\right)\right]$, neither of which change sign. Again we impose an obvious limit $\frac{l^{\prime}}{v_{1}}+\tau_{1} \leqq \frac{2 \pi}{\omega_{2}}$, on $l^{\prime}$ and the duration $\tau_{1}$ of the initial turn by player 1 , related to the initial $\tilde{\theta}$ by:

$$
\bar{\theta}=\bar{\theta}_{f}+\omega_{2}\left(\frac{l^{\prime}}{v_{1}}+\tau_{1}\right) \mp \omega_{1} \tau_{1} \quad(\bmod 2 \pi) .
$$

The final two cases $\bar{i}$ and $\vec{j}$ arise only with a near miss by player 2 on player 1 . Here $\dot{\bar{y}}_{f}=0, V_{\bar{\theta}_{f}}=0, V_{\bar{x}_{f}}=0$ and $V_{\bar{\theta}_{f}}=-1$, so that $\bar{A}_{f}=0$. Also $u_{1}=-\operatorname{sgn} V_{\bar{\theta}_{f}}=$ $+\operatorname{sgn}\left(\dot{V}_{\bar{\theta}_{f}}\right)=-\operatorname{sgn} \sin \bar{\theta}_{f}=\mp 1$ for cases $\left\{\begin{array}{l}\bar{i} \\ \bar{j}\end{array}, V_{\bar{x}}\right.$ and $\bar{A}$ remain zero along the final singular arc by player 2 , so that on arrival at the singular arc $V_{\bar{\theta}}^{\prime}=-\bar{x}^{\prime}$, and the prior history of $-V_{\bar{\theta}}$ and $-\bar{A}$ is again easily constructible.

In all the near miss cases described in this section the initial position $(x, y)$ of player 2 , in the coordinate system attached to player 1 , is easily determined as a function of $\theta$ and the parameter indicated in Table 2. The resulting arcs, for cases $\mathrm{a}-\mathrm{d}, \overrightarrow{\mathrm{a}}-\overline{\mathrm{d}}$, and $\mathrm{e}-\mathrm{h}$ in the "plane" $\theta$ are sections of semipermeable pieces of surface which may form part of the separating surface $\Sigma$. The arcs for cases $\overline{\mathrm{e}}-\overline{\mathrm{h}}$ and $\overline{\mathrm{i}}, \overline{\mathrm{j}}$ are candidate pieces for sections of the outer surface $\Sigma^{\prime}$ separating escape by player 1 from victory for player 2 .

\section{Synthesis of the Separating Surface $\Sigma$ in a Numerical Case}

We assume for our example that $v_{1}=\omega_{1}=1, v_{2}=3 / 4, \omega_{2}=3, l_{1}=2$ and $l_{2}=\infty$. The infinite $l_{2}$ eliminates the outer surface $\Sigma^{\prime}$. The separating surface $\Sigma$ is obtained by looking at the sections, for various $\theta$, of all the semi-permeable pieces of surface described in sections 3 and 4 . However, for the example chosen, cases $\mathrm{e}-\mathrm{h}$ (and their left-right reflections) do not arise, while cases $\overline{\mathrm{e}}-\overline{\mathrm{h}}$ occur only at infinite separation, being associated with $\Sigma$. We need, then, only cases $1-7$, $\mathrm{a}-\mathrm{d}, \overline{\mathrm{a}}-\overline{\mathrm{d}}$, and their left-right reflections. It turns out, moreover, that cases $\overline{\mathrm{a}}-\overline{\mathrm{d}}$ are not necessary to the construction of $\Sigma$.

The section corresponding to $\theta=\frac{\pi}{3}$ is shown in Fig. 5 , without cases $\bar{a}-\tilde{d}$ and $\overline{\mathrm{a}}^{\prime}-\overrightarrow{\mathrm{d}}^{\prime}$, in Fig. 5 , with the section of region $\mathscr{R}_{1}$ shaded. The section of the terminal set $T_{1}$ is just the $y$-axis between 0 and +2 . The composite surface $\Sigma$ is made up of semi-permeable pieces which, because of the direction of the adjoint 
vector $\left(V_{x}, V_{y}, V_{\theta}\right)$, prevent player 2 from moving the state into $\mathscr{R}_{1}$ if player 1 plays optimally in the neighborhood of $\Sigma$, and prevent player 1 from moving the state into $\mathscr{R}_{1}$ if player 2 plays optimally in the neighborhood of $\Sigma$.

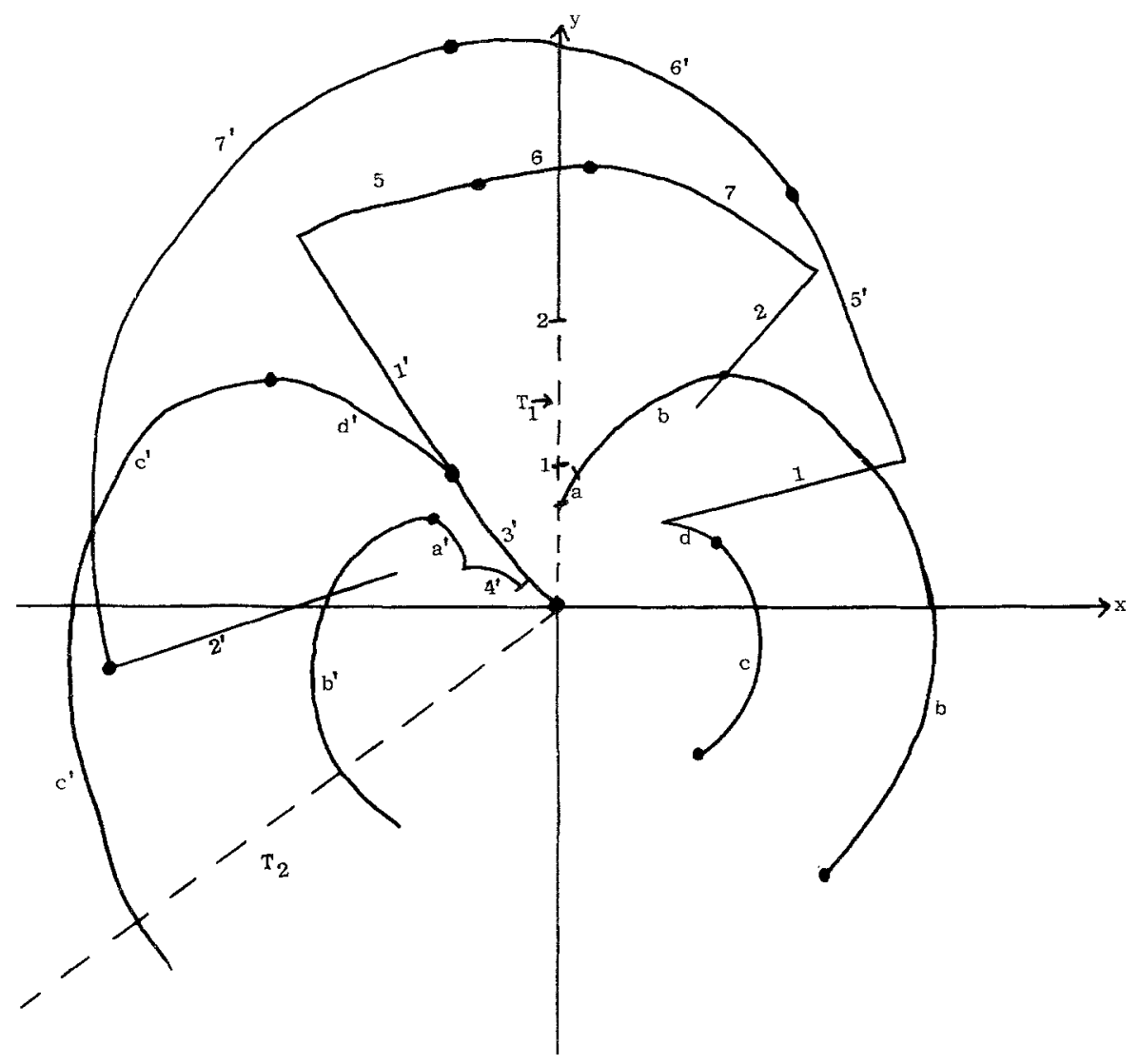

Fig. 5. Complete Section for $\theta=\frac{\pi}{3}$ (without $\overline{\mathrm{a}}-\overline{\mathrm{d}}, \overline{\mathrm{a}}^{\prime}-\overline{\mathrm{d}}^{\prime}$ )

The demonstration of a region $\mathscr{R}_{1}$, entirely surrounded by $T_{1}$ and a composite semi-permeable surface $\Sigma$, requires, of course, the construction of the sections for all values of $\theta$. This has been carried out, nevertheless, for a sufficient number of sections that the region $\mathscr{R}_{1}$ and its boundary are clearly discernible.

Note that in Fig. 5, corresponding to $\theta=\frac{\pi}{3}$, there is a region outside of $\mathscr{R}_{1}$, bounded by $3^{\prime}, d^{\prime}, c^{\prime}, 7^{\prime}, 6^{\prime}, 5^{\prime}, 1$ and $b$. Player 1 can prevent the state from crossing this boundary in a direction away from $\mathscr{R}_{1}$. However, sections for larger $\theta$ show that this region contains part of the terminal set $T_{2}$. The existence of this particular region, if it turns out to be closed, is thus of no significance. 


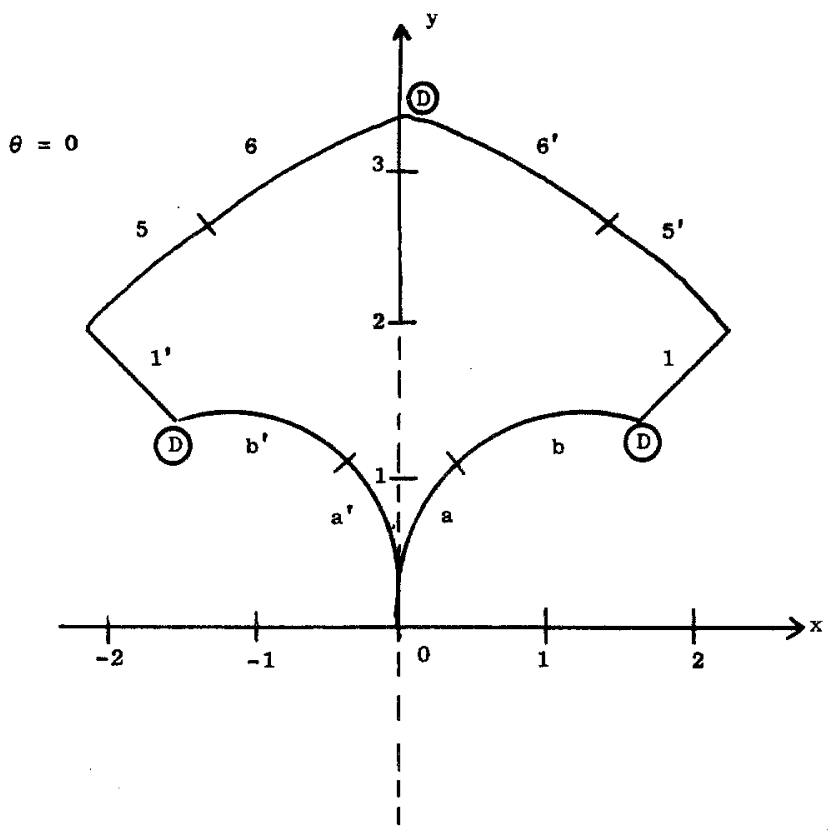

Fig. 6-1

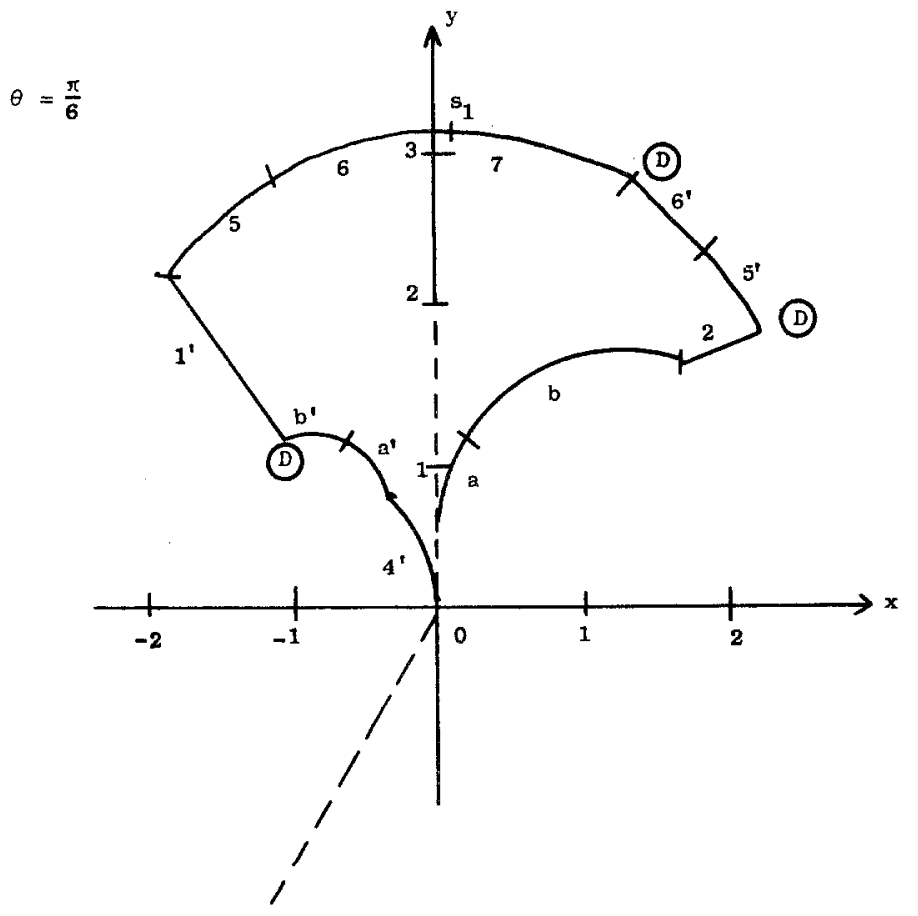

Fig. 6-2 


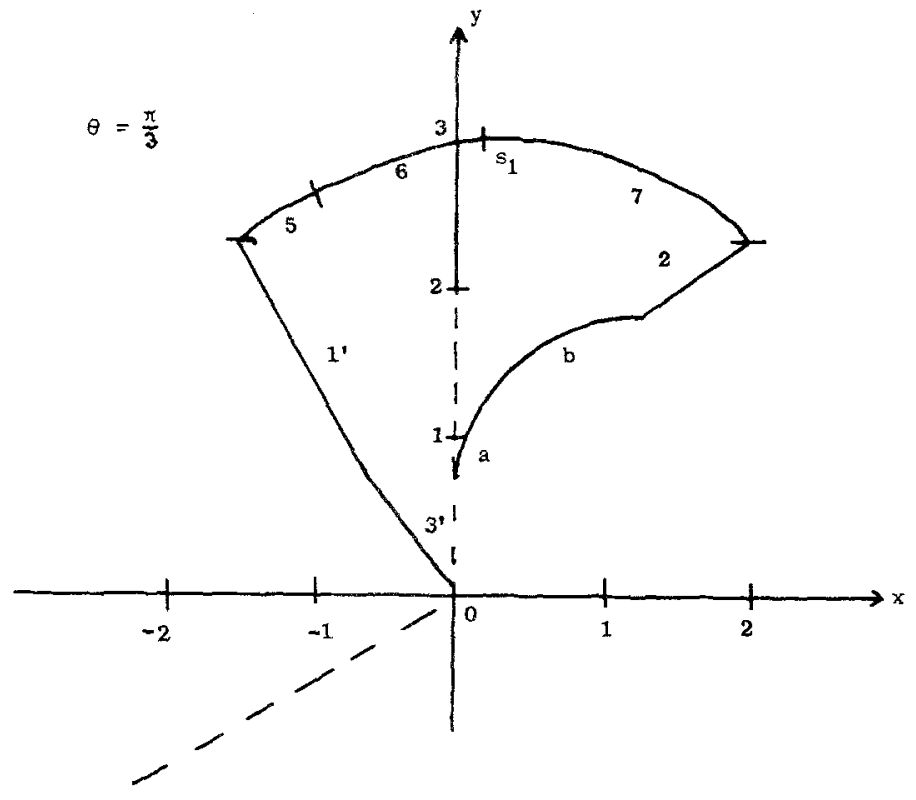

Fig. 6-3

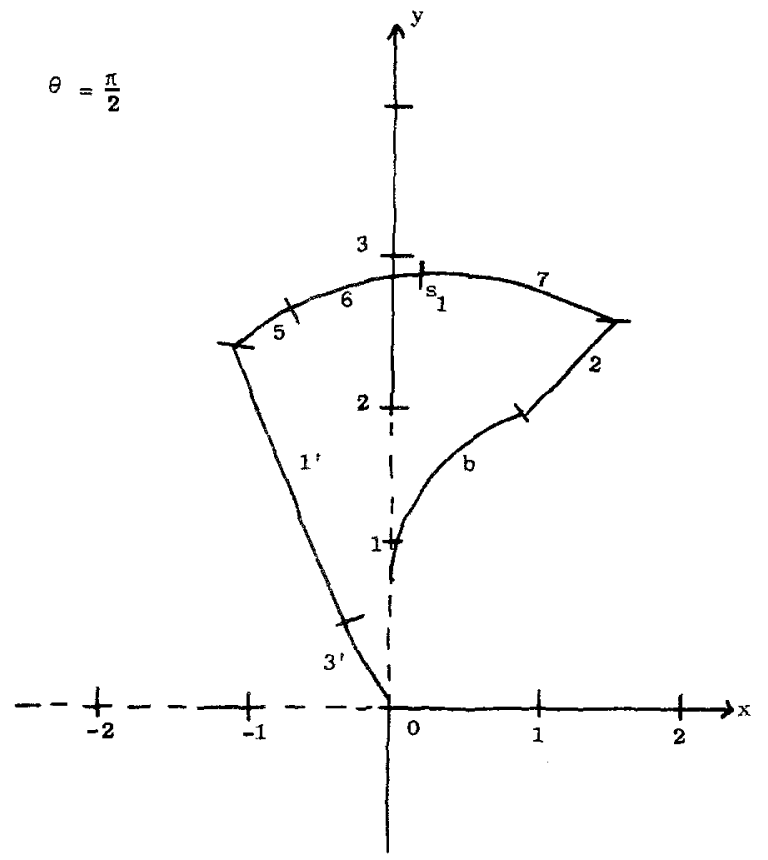

Fig. 6-4 


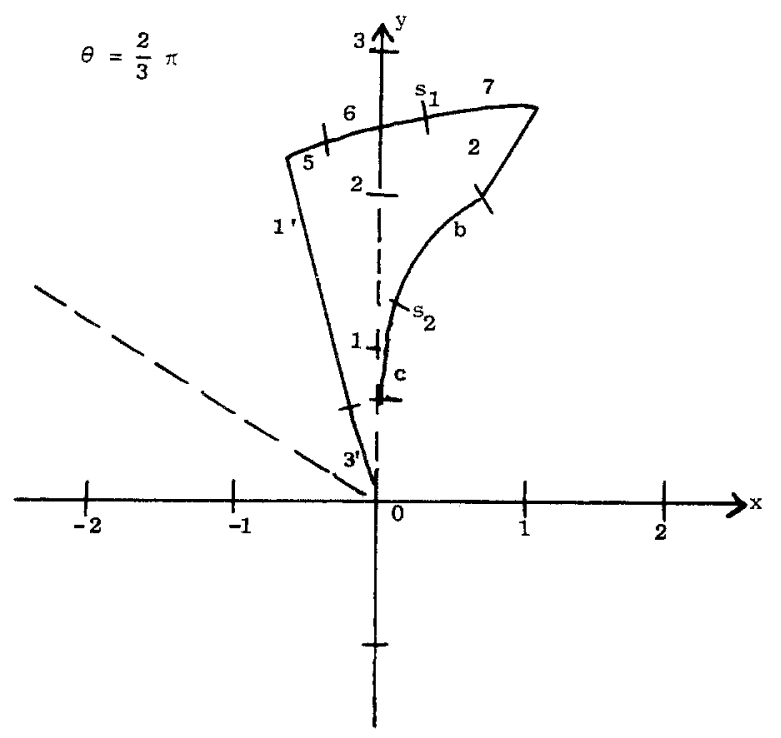

Fig. 6-5

Figs. 6(a) trough 6(e) show certain sections of the region $\mathscr{R}_{1}$ with all unnecessary arcs deleted. The corners of the various sections correspond to edges on the surface $\Sigma$ which are found to be of two types: (i) edges which are followed in optimal play, and (ii) edges, marked (D), which are "dispersal lines" from which the optimal paths move away in two different directions, the choice lying with player 2 who has the larger turn-rate.

P. BERNHARD [1971] has discussed the conditions under which two single semi-permeable surfaces can join to form a composite semi-permeable surface. He requires that the optimal paths either do not approach the edge from either side, or, if they do, they do so either tangentially or with a switch on arrival, so as not to cross the edge. Our edges clearly meet his condition. (See also IsAAcs [1964] p. 267).

Note that the junctions of pieces 5 and 6,6 and $7, a$ and $b, b$ and $c, c$ and $d$, are smooth with continuous adjoint vector (normal).

Finally, Figs. 7 (a) and 7(b) show the projection of the lower and upper portions of the surface $\Sigma$ onto the $x \theta$ "plane", The dashed lines marked $s_{1}$ and $s_{2}$ are the singular arcs corresponding to straight-line motion by player 1 and 2 .

The direction of turn by the two players, when they are turning, is found in all cases to satisfy $u_{1}=-\operatorname{sgn} \dot{x}, u_{2}=+\operatorname{sgn} \dot{\theta}$. 


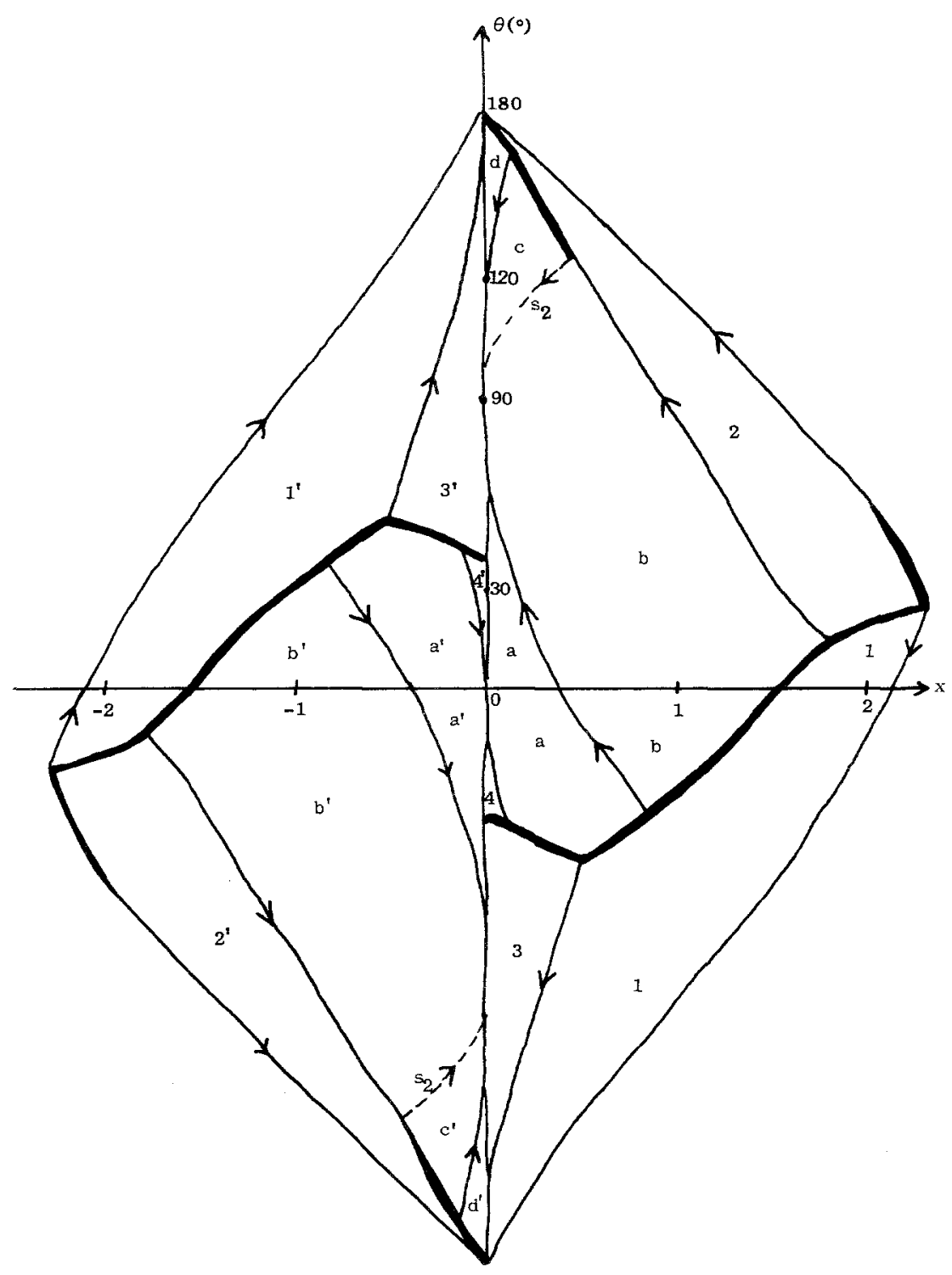

Fig. 7-a 


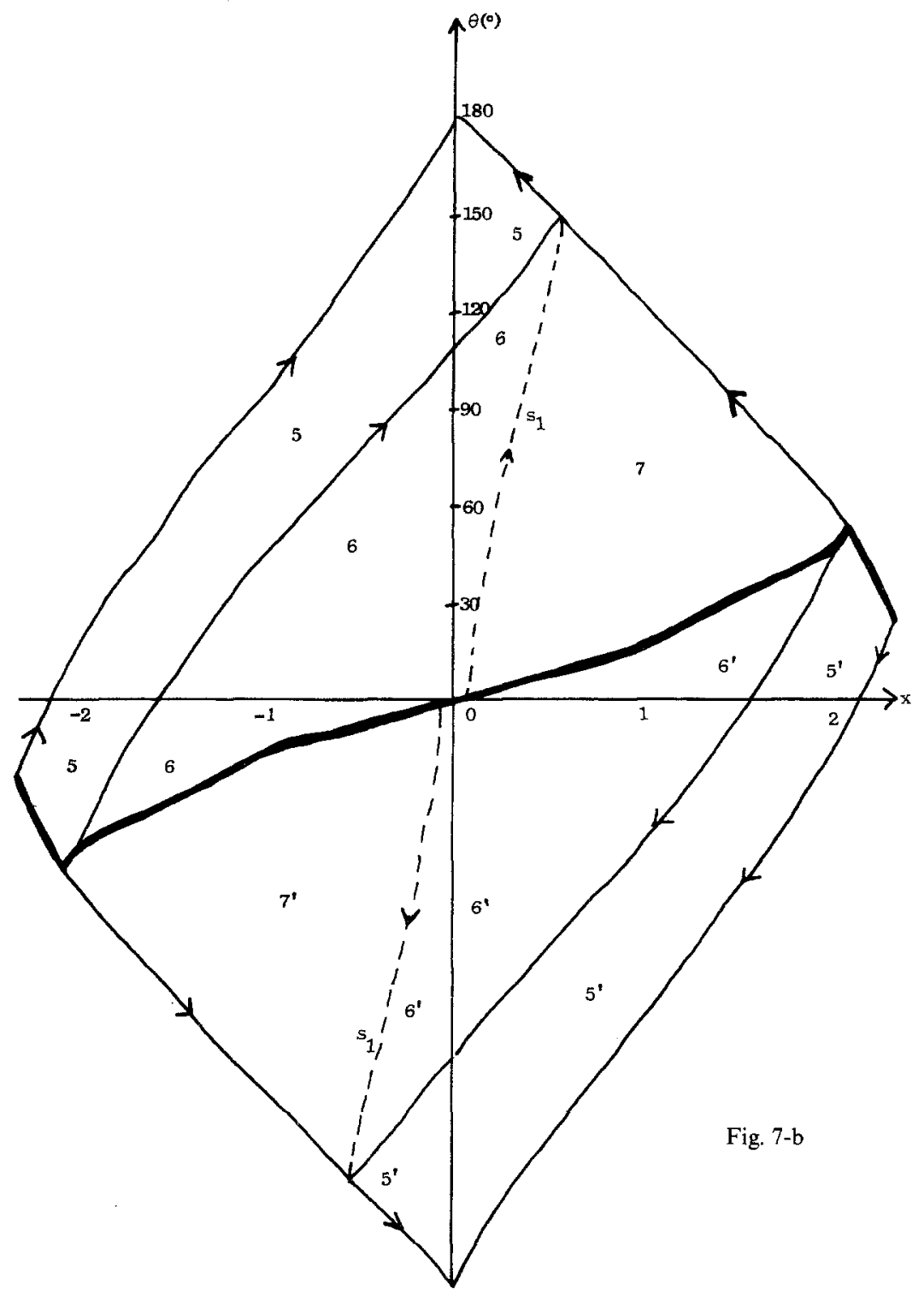

\section{References}

BARoN, S., Y.-C. Ho and D. L. KLEINMANN: A new approach to aerial combat games, NASA CR-1626, 1970 .

Bernhard, P.: Conditions de coin pour les jeux differentiels, Seminaire sur les Jeux Differentiels, Centre D'Automatique, Paris, 1971.

Ciletti, M. D., L. Meier and D. M. SALmon: Applications of reachable sets techniques to air combat analysis, A.I. A. A. 11th Aerospace Sciences Meeting, Washington, D. C., January 1973.

Falco, M., and V. CoHEN: Strategy synthesis in aerial dogfight, A.I.A. A. 11th Aerospace Sciences Meeting, Washington, D. C., January 1973.

IsAACs, R.: Differential Games, Wiley 1964.

Received November, 1973. 